\title{
Neutrophil-to-Lymphocyte Ratio Predicts Early Mortality in Patients with HBV-Related Decompensated Cirrhosis
}

\author{
Honggang Zhang, ${ }^{1}$ Qinqin Sun, ${ }^{2}$ Weilin Mao, ${ }^{3}$ Jian Fan, ${ }^{3}$ and Bo $\mathrm{Ye}^{3}$ \\ ${ }^{1}$ Department of Anesthesiology, The First Affiliated Hospital, College of Medicine, Zhejiang University, Zhejiang 310003, China \\ ${ }^{2}$ Department of Urology, The Sixth Affiliated Hospital of Xinjiang Medical University, Xinjiang 830001, China \\ ${ }^{3}$ Department of Clinical Laboratory, The First Affiliated Hospital, College of Medicine, Zhejiang University, Zhejiang 310003, China
}

Correspondence should be addressed to Bo Ye; wenyiabo@163.com

Received 28 October 2015; Revised 30 December 2015; Accepted 3 January 2016

Academic Editor: Edoardo G. Giannini

Copyright (C) 2016 Honggang Zhang et al. This is an open access article distributed under the Creative Commons Attribution License, which permits unrestricted use, distribution, and reproduction in any medium, provided the original work is properly cited.

\begin{abstract}
Background. The neutrophil-to-lymphocyte ratio (NLR) is an inflammation index that has been shown to independently predict poor clinical outcomes. We aimed to evaluate the clinical value of NLR in the prediction of 30-day mortality in patients with HBVrelated decompensated cirrhosis (HBV-DeCi). Methods. This was a retrospective cohort study that included 148 patients with HBVDeCi. Results. An elevated NLR was associated with increased severity of liver disease and mortality within 30 days. Multivariate analysis suggested that NLR, similar to the model for end-stage liver disease (MELD) score, is an additional independent predictor of 30-day mortality $(P<0.01)$. Conclusion. Our results suggest that a high NLR can be considered a new independent biomarker for predicting 30-day mortality in patients with HBV-DeCi.
\end{abstract}

\section{Introduction}

All chronic liver diseases can lead to liver cirrhosis (LC). Hepatic decompensation ultimately occurs in cirrhotic patients as a result of progressive portal hypertension, impairment of hepatic biologic functions, or cancer development [1, 2]. Chronic hepatitis B virus (HBV) infection remains a major cause of LC in China, with a yearly incidence of decompensated cirrhosis (DeCi) of 3\% [3]. Previous studies have shown that the prognosis of $\mathrm{DeCi}$ is usually poor with a 5 -year survival rate of only $14-35 \%$ under conventional standard care $[4,5]$. Although the majority of patients with HBV-related decompensated cirrhosis (HBV-DeCi) can be referred for liver transplantation, the shortage of donor livers and considerable cost make this approach unavailable for most patients at present $[6,7]$. Therefore, discovery of a marker associated with HBV-DeCi severity will help improve clinical management to mitigate the high rate of mortality.

Necroinflammation is an essential component of liver pathology in chronic HBV infection. The neutrophil-tolymphocyte ratio (NLR), a simple and effective marker that reflects the severity of inflammation, is easily calculated from routinely available data. Neutrophilia occurs in chronic inflammation, and lymphopenia is associated with malnutrition and bacterial infection. High NLRs have been shown to predict outcomes in various disease processes including cardiac disease, malignancy, and renal failure [8-10]. More recently, Chen et al. and Liu et al. showed that the NLR measured on hospital admission can serve as an independent predictor of the 3-month mortality rate in patients with acute-on-chronic liver failure (AoCLF) [11, 12]. Furthermore, Biyik et al. found that a greater NLR is associated with an increased risk of long-term death in cirrhotic patients who are at an early advanced stage (mean model for end-stage liver disease (MELD) score 10 and Child-Pugh score 7) [13]. However, currently there are few markers that can predict 30day mortality after hospital admission of patients with DeCi. In the present study, we hence investigated NLR as a predictor for 1-month mortality in a cohort of HBV-DeCi patients.

\section{Patients and Methods}

This was a retrospective follow-up study of a cohort of 148 consecutive in-patients who were diagnosed with 
decompensated cirrhosis and met the inclusion and exclusion criteria between January 2014 and January 2015.

For inclusion, patients had to be HBsAg positive, previously diagnosed with $\mathrm{HBV}$-related compensated cirrhosis, and presenting clinical manifestations of decompensated liver disease for the first time. Patients were excluded according to the following criteria: acute hepatitis; hematologic disorder; malignancy such as hepatocellular carcinoma; pregnancy; concurrent infection with $\mathrm{HCV}$, hepatitis D virus, or human immunodeficiency virus; or concurrent autoimmune or other liver diseases.

Decompensation was defined by the appearance of clinical ascites, variceal bleeding, jaundice, or hepatic encephalopathy (HE) [14]. At baseline, for each patient, demographic and clinical data including age, sex, and complications of ascites, variceal bleeding, hepatorenal syndrome (HRS), or HE and clinical course in the hospital were obtained from the medical files and recorded in a specified liver disease pro forma. In our cohort, 120 patients were receiving antiviral therapy, 70 had started antiviral therapy before admission, and 50 had started after admission. Only 28 patients did not receive any antiviral therapy throughout the clinical course for economic reasons. Biochemical parameters including creatinine, liver function tests, white cell and differential counts, and platelet counts were recorded. The NLR was calculated as the absolute neutrophil count divided by the absolute lymphocyte count. A NLR $\geq 5$ was considered elevated $[15,16]$. In addition, the MELD score and serological indexes (HBsAg, HBeAg, anti-HBc, and HBV DNA levels) were detected at baseline.

The study was performed in accordance with the Declaration of Helsinki, and the procedures were approved by the Ethics Committee of the First Affiliated Hospital of Zhejiang University College of Medicine. The need for written patient consent was waived.

2.1. MELD Score. Liver disease severity was evaluated using the MELD score, which includes the patient's serum bilirubin and creatinine levels and the international normalized ratio (INR) for prothrombin time. The MELD score was calculated using an online calculator (http://www .mayoclinic.org/gi-rst/mayomodel7.html).

2.2. Statistical Analysis. All continuous variables were expressed as mean \pm standard deviation (SD) or median (range), and categorical data were calculated as percentages. The differences in the variables were weighted using analysis of variance (ANOVA) or the Kruskal-Wallis tests. Categorical data were evaluated by the $\chi^{2}$-test, as appropriate. Correlations between variables were examined using Spearman's correlation test. Multivariate analysis was performed using Cox proportional hazards regression that weighed all possible clinical factors. The receiver operator characteristic (ROC) curves and the respective areas under the curve (AUCs) were used to assess the ability for the prediction of death. Statistical analyses were performed using the SPSS version 12.0 statistical package (SPSS Inc.,
TABLE 1: Baseline demographic and clinical characteristics of cohort.

\begin{tabular}{lc}
\hline & HBV-DeCi patients $(n=148)$ \\
\hline Gender $($ male/female $)$ & $118 / 30$ \\
Age $(\mathrm{y})$ & $53.2 \pm 11.2$ \\
Total protein $(\mathrm{g} / \mathrm{L})$ & $61.0 \pm 8.2$ \\
Albumin $(\mathrm{g} / \mathrm{L})$ & $30.4 \pm 5.9$ \\
ALT $(\mathrm{U} / \mathrm{L})$ & $30.5(17.0-55.0)$ \\
AST $(\mathrm{U} / \mathrm{L})$ & $48.0(29.0-79.0)$ \\
Total bilirubin $(\mu \mathrm{mol} / \mathrm{L})$ & $50.5(23.5-117.0)$ \\
INR & $1.50 \pm 0.40$ \\
Creatinine $(\mathrm{mmol} / \mathrm{L})$ & $72.5(60.0-88.0)$ \\
Platelet count $\left(\times 10^{9} / \mathrm{L}\right)$ & $65.5(38.0-113.5)$ \\
White blood cell count $\left(\times 10^{9} / \mathrm{L}\right)$ & $4.35(2.90-5.90)$ \\
Neutrophil count $\left(\times 10^{9} / \mathrm{L}\right)$ & $2.40(1.50-3.70)$ \\
Lymphocyte count $\left(\times 10^{9} / \mathrm{L}\right)$ & $0.90(0.60-1.40)$ \\
NLR & $2.67(1.46-4.39)$ \\
MELD score & $13.4 \pm 6.7$ \\
HBsAg positive & 148 \\
HBeAg positive & 80 \\
HBcAb IgM positive & 0 \\
HBV DNA positive & 148 \\
HE $(n)$ & 3 \\
Ascites $(n)$ & 107 \\
Variceal bleeding $(n)$ & 40 \\
HRS $(n)$ & 29 \\
\hline & $3 a$ a $)$ \\
\hline
\end{tabular}

Data are expressed as $n$, mean $\pm \mathrm{SD}$, or median (interquartile range).

ALT, alanine aminotransferase; AST, aspartate aminotransferase; INR, international normalized ratio; NLR, neutrophil-to-lymphocyte ratio; MELD score, model for end-stage liver disease score; HE, hepatic encephalopathy; HRS, hepatorenal syndrome.

Chicago, IL), and a $P<0.05$ was considered statistically significant.

\section{Results}

3.1. Baseline Characteristics of All Patients. A total of 148 patients (118 males, mean age: $53.2 \pm 11.2$ years) with HBVDeCi were enrolled in the present study. Their baseline characteristics are summarized in Table 1. A significant positive correlation between NLR and MELD score $(r=0.241, P=$ 0.003 ) was detected in patients (Figure 1).

3.2. Baseline Characteristics of Patients with Different NLR Levels. HBV-DeCi patients were divided into three groups based on NLR levels: group A $(\mathrm{NLR} \leq 2.0)$, group B $(>2.0$, but $<5.0)$, and group $C(\geq 5.0)$. The clinical and laboratory characteristics of the three groups are listed in Table 2 . There were significant differences in total protein, serum albumin, serum creatinine, MELD score, and mortality rate among the three groups $(P=0.004, P=0.023, P=0.013, P=0.033$, and $P=0.036$, resp.). Moreover, highly elevated NLR levels were associated with higher frequencies of clinical complications such as HRS $(P=0.035)$. No significant differences in ALT, AST, total bilirubin, INR levels, gender, or age were detected. 
TABLE 2: Clinical and laboratory characteristics in patients with different neutrophil-to-lymphocyte ratio (NLR) at admission.

\begin{tabular}{|c|c|c|c|c|}
\hline & $\begin{array}{c}\text { Group A } \\
(\mathrm{NLR} \leq 2.0, n=61)\end{array}$ & $\begin{array}{c}\text { Group B } \\
(2.0<\mathrm{NLR}<5.0, n=56)\end{array}$ & $\begin{array}{c}\text { Group C } \\
(\mathrm{NLR} \geq 5.0, n=31)\end{array}$ & $P$ \\
\hline Age (y) & $53.0 \pm 10.1$ & $53.5 \pm 12.7$ & $52.8 \pm 10.7$ & 0.946 \\
\hline Gender (male/female) & $47 / 14$ & $47 / 9$ & $24 / 7$ & 0.611 \\
\hline Total protein $(\mathrm{g} / \mathrm{L})$ & $61.7 \pm 6.4$ & $62.6 \pm 8.0$ & $56.8 \pm 10.4$ & 0.004 \\
\hline Albumin (g/L) & $29.6 \pm 5.9$ & $32.0 \pm 5.9$ & $28.9 \pm 5.4$ & 0.023 \\
\hline $\operatorname{ALT}(\mathrm{U} / \mathrm{L})$ & $44.5 \pm 44.1$ & $59.4 \pm 93.2$ & $65.2 \pm 72.5$ & 0.379 \\
\hline AST (U/L) & $57.5 \pm 39.0$ & $81.0 \pm 118.4$ & $94.6 \pm 98.5$ & 0.182 \\
\hline INR & $1.46 \pm 0.29$ & $1.50 \pm 0.48$ & $1.56 \pm 0.41$ & 0.471 \\
\hline Creatinine $(\mathrm{mmol} / \mathrm{L})$ & $74.9 \pm 19.9$ & $77.4 \pm 43.4$ & $100.4 \pm 61.1$ & 0.013 \\
\hline Total bilirubin $(\mu \mathrm{mol} / \mathrm{L})$ & $79.8 \pm 91.5$ & $98.3 \pm 107.8$ & $123.3 \pm 162.9 .37$ & 0.233 \\
\hline MELD score & $12.46 \pm 1.73$ & $13.88 \pm 6.15$ & $16.14 \pm 7.84$ & 0.033 \\
\hline White blood cell count $\left(\times 10^{9} / \mathrm{L}\right)$ & $3.51 \pm 6.22$ & $4.62 \pm 2.09$ & $10.00 \pm 8.66$ & $<0.001$ \\
\hline Neutrophil count $\left(\times 10^{9} / \mathrm{L}\right)$ & $1.63 \pm 0.79$ & $3.00 \pm 1.38$ & $8.22 \pm 7.36$ & $<0.001$ \\
\hline Lymphocyte count $\left(\times 10^{9} / \mathrm{L}\right)$ & $1.33 \pm 0.71$ & $0.95 \pm 0.45$ & $0.88 \pm 0.81$ & 0.001 \\
\hline Ascites (yes/no) & $40 / 21$ & $45 / 11$ & $22 / 9$ & 0.200 \\
\hline HRS (yes/no) & $8 / 53$ & $10 / 46$ & $11 / 20$ & 0.035 \\
\hline HE (yes/no) & $0 / 61$ & $1 / 55$ & $2 / 29$ & - \\
\hline Mortality (yes/no) & $3 / 58$ & $6 / 50$ & $7 / 24$ & 0.036 \\
\hline
\end{tabular}

Data are expressed as $n$ or mean \pm SD.

ALT, alanine aminotransferase; AST, aspartate aminotransferase; INR, international normalized ratio; NLR, neutrophil-to-lymphocyte ratio; MELD score, model for end-stage liver disease score; HE, hepatic encephalopathy; HRS, hepatorenal syndrome.

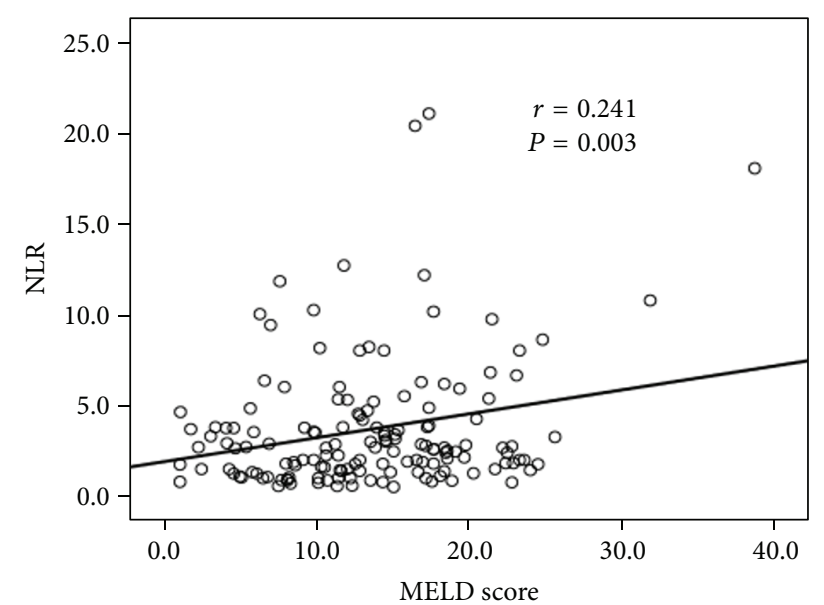

FIGURE 1: Scatter diagram for correlation analysis, showing a positive correlation between the NLR and MELD score in HBV-DeCi patients.

Furthermore, patients in group $\mathrm{C}$ had significantly higher white cell and neutrophil counts than patients in groups $\mathrm{A}$ and $\mathrm{B}$, whereas the lymphocyte count was lower than those in groups A and B. These data suggest that a higher NLR in DeCi patients could be primarily attributed to increased neutrophil counts and decreased lymphocyte counts.

3.3. Relative Risk Factors for 30-Day Mortality. The patients were followed up for a median of 20 days (IQR: $13-80$ days). During the follow-up, 16 patients died within 1 month due to upper gastrointestinal bleeding $(n=6), \mathrm{HE}(n=3)$, or HRS $(n=7)$. Our findings indicated that an elevated NLR level at admission was followed by an increased 1-month mortality rate, increasing from $4.9 \%$ in group A to $10.7 \%$ in group B and $22.6 \%$ in group C. Univariate and multivariate logistic regression analyses showed that a high NLR was an additional independent risk factor for 1-month mortality in $\mathrm{HBV}-\mathrm{DeCi}$ patients, similar to a high MELD score (Table 3). To evaluate the ability of NLR and MELD scores to predict mortality, ROC curves were obtained (Figure 2). The AUC values were $0.832 \pm 0.062$ for the MELD score and $0.736 \pm 0.076$ for NLR (both $P<0.005$ ). When NLR and MELD score were combined, the AUC was $0.891 \pm 0.057(P<0.001)$. The sensitivity and specificity of the MELD score were $91.2 \%$ and $73.4 \%$, respectively, with $29.8 \%$ positive values and $98.5 \%$ negative predictive values. When the NLR and MELD score were combined, the sensitivity $(93.8 \%)$ and specificity $(81.0 \%)$ were significantly improved, with $37.9 \%$ positive values and $99.1 \%$ negative predictive values.

\section{Discussion}

In the present study, we examined the ability of the blood NLR, a simple marker that reflects the severity of inflammation, to predict 1-month mortality in HBV-DeCi patients. We made two important observations. First, we found that a higher NLR correlated with increased frequency of liver related complications such as HRS. Secondly, we demonstrated that an elevated NLR level can serve as an independent predictor for mortality in HBV-DeCi patients and the risk of mortality increases as the NLR increases. 
TABLE 3: Cox proportional hazards analysis for predictors of mortality.

\begin{tabular}{|c|c|c|c|c|c|c|}
\hline & \multicolumn{3}{|c|}{ Univariate } & \multicolumn{3}{|c|}{ Multivariate } \\
\hline & Odds ratio & $95 \% \mathrm{CI}$ & $P$ & Odds ratio & $95 \% \mathrm{CI}$ & $P$ \\
\hline NLR & 1.226 & $1.071-1.402$ & 0.003 & 1.296 & $1.047-1.604$ & 0.017 \\
\hline MELD score & 1.245 & $1.115-1.391$ & $<0.001$ & 1.274 & $1.031-1.573$ & 0.025 \\
\hline Age (y) & 1.003 & $1.000-1.051$ & 0.896 & - & - & 0.917 \\
\hline Albumin (g/L) & 0.995 & $0.908-1.090$ & 0.442 & - & - & 0.180 \\
\hline Total protein $(\mathrm{g} / \mathrm{L})$ & 0.996 & $0.930-1.067$ & 0.910 & - & - & 0.604 \\
\hline White blood cell count $\left(\times 10^{9} / \mathrm{L}\right)$ & 1.031 & $0.900-1.118$ & 0.663 & - & - & 0.223 \\
\hline
\end{tabular}

CI, confidence interval; NLR, neutrophil-to-lymphocyte ratio; MELD score, model for end-stage liver disease score.

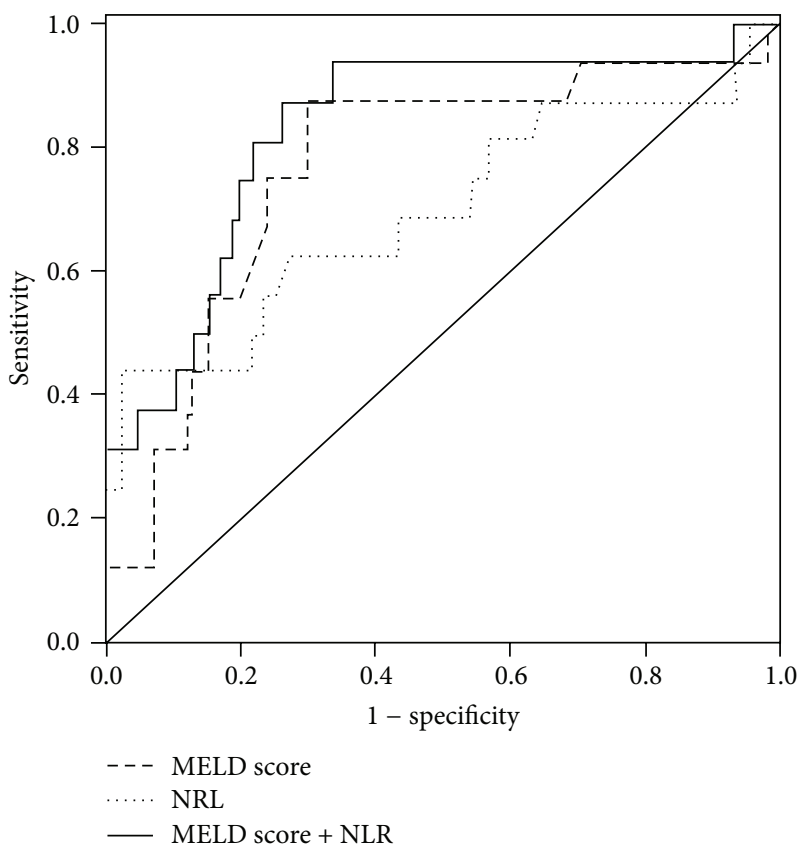

FIGURE 2: Receiver operating characteristic curve, indicating the relative efficiencies for prediction of 1-month mortality by $\operatorname{NLR}(\ldots)$, MELD score (- - ), and their combination (-).

The MELD score is known to signal risk for 3-month mortality and is used to assign priorities in the transplant waiting list for cadaveric livers [17]. Our previous study reported that the MELD score was associated with the prognosis of patients with AoCLF [18]. In the present retrospective study of HBV$\mathrm{DeCi}$, a significant positive association was found between the NLR and the MELD score. The NLR can be used to predict HBV-DeCi patients' mortality, although the predictive power of NLR was relatively lower than that of the MELD score. However, the NLR involves only two markers, which makes it simpler and easier to calculate than the MELD score. A combination of NLR with the MELD score augmented the predictive power.

The physiopathologic association between an elevated NLR and poor prognosis is complex and remains to be elucidated. Chronic HBV infection can cause ongoing liver injury. Repeated repair of the damaged liver parenchyma could lead to fibrosis and cirrhosis. A cirrhosis patient who has advanced to the decompensatory stage can be very vulnerable to new insults including reactivation of $\mathrm{HBV}$ replication in the infected liver. Elevated neutrophil counts are a key component of an increased NLR. In our study, we noted that there were marked increases in the white cell and neutrophil counts with a higher NLR compared with those with lower NLR levels. We reasoned that newly intensified hepatic inflammation in DeCi patients during flare-ups of liver injury triggered a large neutrophil response. In addition, lymphocyte counts were lower with a higher NLR compared with those with lower NRLs in our study. One possible explanation for the reduced number of lymphocytes in peripheral blood is that lymphocytes were largely recruited to the liver to participate in the necroinflammation. A previous investigation showed that the intrahepatic CD8+ T-cell number was approximately 50-fold greater in AoCLF patients compared to normal individuals [19]. Lymphopenia was once an indication of malnutrition and poor response of immunity in patients with chronic liver disease [20, 21]. Our results were in accordance with those of Berres et al., who found that a lower lymphocyte count was associated with mortality in patients with end-stage cirrhosis listed for liver transplantation [22]. Our findings indicate that the higher NLR mainly resulted from increased neutrophil counts and decreased lymphocyte counts. Therefore, a high neutrophil count together with a low lymphocyte count may reflect the severity and progress of liver injury in HBV-DeCi patients.

\section{Conclusion}

This study of the NLR in hospitalized patients with HBVDeCi showed that an elevated NLR was associated with the severity of HBV-DeCi, and the NLR can function as an additional marker for predicting 1-month mortality in this cohort. However, possible application of our findings is limited by the retrospective nature of the study and the relatively small number of patients included. Clearly, further prospective studies are warranted to confirm our findings.

\section{Abbreviations}

HBV: Hepatitis B virus

CI: Confidence interval

DeCi: Decompensated cirrhosis 


$\begin{array}{ll}\text { NLR: } & \text { Neutrophil-to-lymphocyte ratio } \\ \text { ALT: } & \text { Alanine aminotransferase } \\ \text { AST: } & \text { Aspartate aminotransferase } \\ \text { MELD score: } & \text { Model for end-stage liver disease score } \\ \text { INR: } & \text { International normalized ratio } \\ \text { HE: } & \text { Hepatic encephalopathy } \\ \text { HRS: } & \text { Hepatorenal syndrome } \\ \text { ROC: } & \text { Receiver operating characteristic } \\ \text { AUCs: } & \text { Areas under the curve. }\end{array}$

\section{Conflict of Interests}

None of the authors have any commercial or other association that might pose a conflict of interests.

\section{References}

[1] M. Y. Kim, S. K. Baik, and S. S. Lee, "Hemodynamic alterations in cirrhosis and portal hypertension," The Korean Journal of Hepatolog, vol. 16, no. 4, pp. 347-352, 2010.

[2] J. W. Jang, "Current status of liver diseases in Korea: liver cirrhosis," Korean Journal of Hepatology, vol. 15, pp. S40-S49, 2009.

[3] E. A. Tsochatzis, J. Bosch, and A. K. Burroughs, "Liver cirrhosis," The Lancet, vol. 383, no. 9930, pp. 1749-1761, 2014.

[4] S.-B. Wang, J.-H. Wang, J. Chen, R. K. Giri, and M.-H. Chen, "Natural history of liver cirrhosis in south China based on a large cohort study in one center: a follow-up study for up to 5 years in 920 patients," Chinese Medical Journal, vol. 125, no. 12, pp. 2157-2162, 2012.

[5] P. M. Harrison, "Management of patients with decompensated cirrhosis," Clinical Medicine, vol. 15, no. 2, pp. 201-203, 2015.

[6] G. E. Chung, J.-H. Lee, and Y. J. Kim, "Does antiviral therapy reduce complications of cirrhosis?" World Journal of Gastroenterology, vol. 20, no. 23, pp. 7306-7311, 2014.

[7] A. C. Chan, S. T. Fan, C. M. Lo et al., "Liver transplantation for acute-on-chronic liver failure," Hepatology International, vol. 3, no. 4, pp. 571-581, 2009.

[8] T. Bhat, S. Teli, J. Rijal et al., "Neutrophil to lymphocyte ratio and cardiovascular diseases: a review," Expert Review of Cardiovascular Therapy, vol. 11, no. 1, pp. 55-59, 2013.

[9] G. Absenger, J. Szkandera, M. Pichler et al., "A derived neutrophil to lymphocyte ratio predicts clinical outcome in stage II and III colon cancer patients," British Journal of Cancer, vol. 109, no. 2, pp. 395-400, 2013.

[10] D. N. Reddan, P. S. Klassen, L. A. Szczech et al., "White blood cells as a novel mortality predictor in haemodialysis patients," Nephrology Dialysis Transplantation, vol. 18, no. 6, pp. 1167-1173, 2003.

[11] L. Chen, Y. Lou, Y. Chen, and J. Yang, "Prognostic value of the neutrophil-to-lymphocyte ratio in patients with acute-onchronic liver failure," International Journal of Clinical Practice, vol. 68, no. 8, pp. 1034-1040, 2014.

[12] H. Liu, H. Zhang, G. Wan et al., "Neutrophil-lymphocyte ratio: a novel predictor for short-term prognosis in acute-on-chronic hepatitis B liver failure," Journal of Viral Hepatitis, vol. 21, no. 7, pp. 499-507, 2014.

[13] M. Biyik, R. Ucar, Y. Solak et al., "Blood neutrophil-tolymphocyte ratio independently predicts survival in patients with liver cirrhosis," European Journal of Gastroenterology and Hepatology, vol. 25, no. 4, pp. 435-441, 2013.
[14] Yun-Fan Liaw, Dar-In Tai, Chia-Ming Chu, and Tong-Jong Chen, "The development of cirrhosis in patients with chronic type B hepatitis: a prospective study," Hepatology, vol. 8, no. 3, pp. 493-496, 1988.

[15] K. J. Halazun, A. Aldoori, H. Z. Malik et al., "Elevated preoperative neutrophil to lymphocyte ratio predicts survival following hepatic resection for colorectal liver metastases," European Journal of Surgical Oncology, vol. 34, no. 1, pp. 55-60, 2008.

[16] S. R. Walsh, E. J. Cook, F. Goulder, T. A. Justin, and N. J. Keeling, "Neutrophil-lymphocyte ratio as a prognostic factor in colorectal cancer," Journal of Surgical Oncology, vol. 91, no. 3, pp. 181-184, 2005.

[17] R. B. Freeman Jr., R. H. Wiesner, A. Harper et al., "The new liver allocation system: moving toward evidence-based transplantation policy," Liver Transplantation, vol. 8, no. 9, pp. 851-858, 2002.

[18] W. Mao, B. Ye, S. Lin, Y. Fu, Y. Chen, and Y. Chen, "Prediction value of model for end-stage liver disease scoring system on prognosis in the acute on chronic liver failure patients with plasma exchange treatment," ASAIO Journal, vol. 56, no. 5, pp. 475-478, 2010.

[19] Z. Zou, D. Xu, B. Li et al., "Compartmentalization and its implication for peripheral immunologically-competent cells to the liver in patients with HBV-related acute-on-chronic liver failure," Hepatology Research, vol. 39, no. 12, pp. 1198-1207, 2009.

[20] S. J. O'Keefe, A. R. El-Zayadi, and T. E. Carraher, "Malnutrition and immuno-incompetence in patients with liver disease," The Lancet, vol. 2, no. 8195, pp. 615-617, 1980.

[21] M. Fernández-Ruiz, F. López-Medrano, E. M. Romo et al., "Pretransplant lymphocyte count predicts the incidence of infection during the first two years after liver transplantation," Liver Transplantation, vol. 15, no. 10, pp. 1209-1216, 2009.

[22] M.-L. Berres, B. Schnyder, E. Yagmur et al., "Longitudinal monocyte Human leukocyte antigen-DR expression is a prognostic marker in critically ill patients with decompensated liver cirrhosis," Liver International, vol. 29, no. 4, pp. 536-543, 2009. 


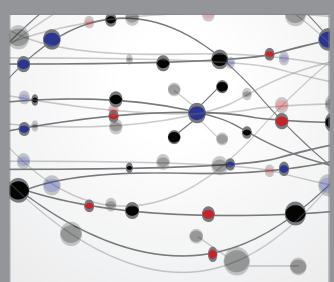

The Scientific World Journal
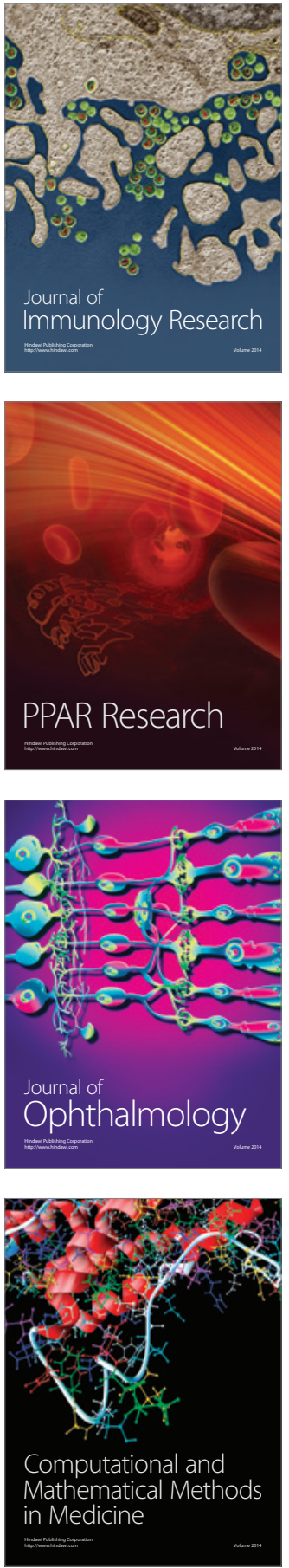

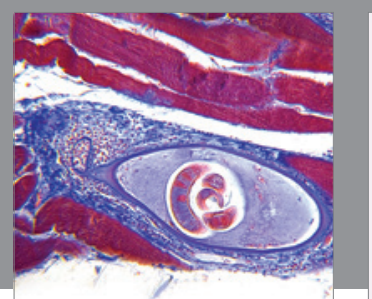

Gastroenterology Research and Practice

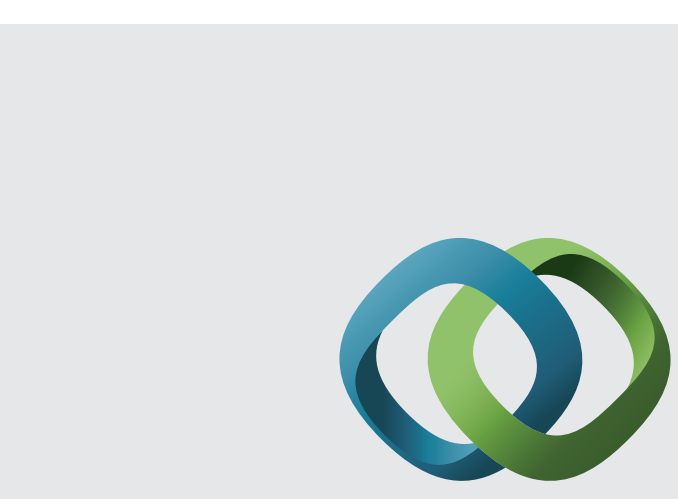

\section{Hindawi}

Submit your manuscripts at

http://www.hindawi.com
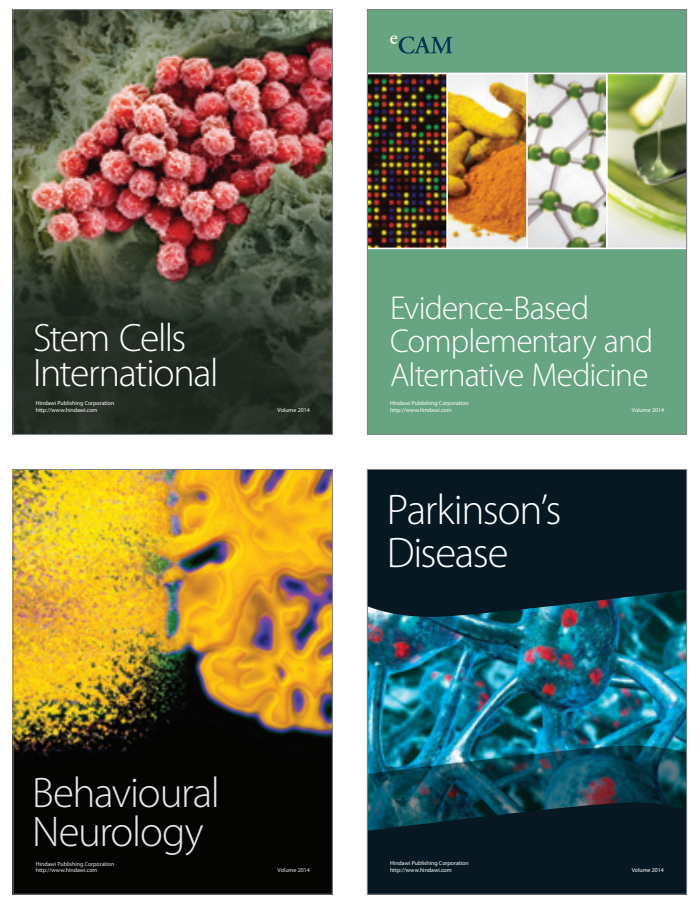
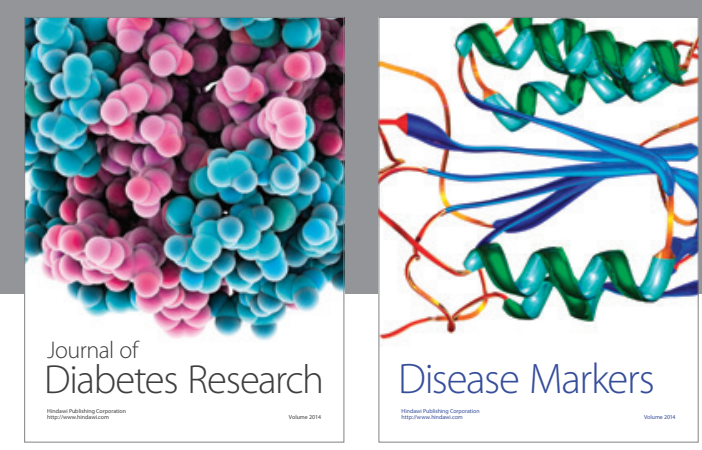

Disease Markers
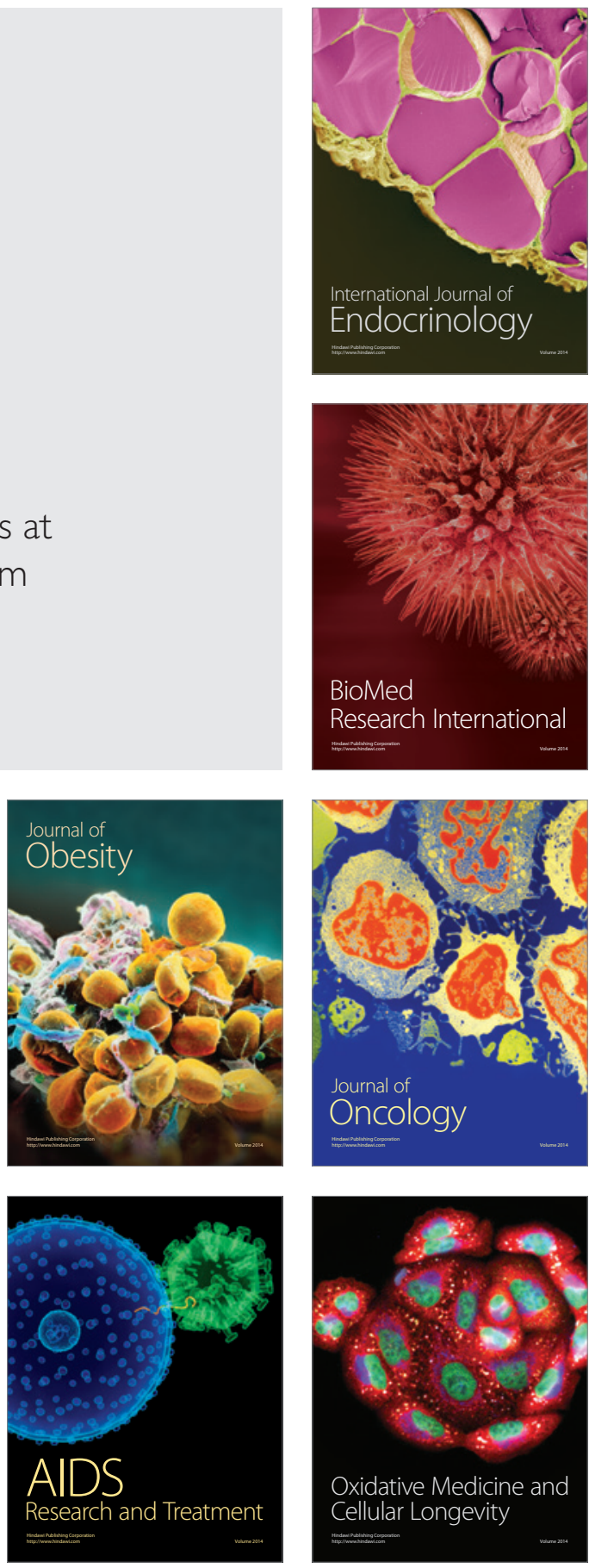